J. Korean Math. Soc. 46 (2009), No. 3, pp. 643-656

DOI 10.4134/JKMS.2009.46.3.643

\title{
DEMI-LINEAR ANALYSIS I-BASIC PRINCIPLES
}

\author{
Ronglu Li, Shuhui Zhong, And Linsong Li
}

\begin{abstract}
The family of demi-linear mappings between topological vector spaces is a meaningful extension of the family of linear operators. We establish equicontinuity results for demi-linear mappings and develop the usual theory of distributions and the usual duality theory.
\end{abstract}

For topological vector spaces $X, Y$ and the family $L(X, Y)$ of continuous linear operators, the classical equicontinuity theorem says that if $X$ is of second category and $\Gamma$ is a pointwise bounded subfamily of $L(X, Y)$, then $\Gamma$ is equicontinuous at each $x \in X$ so $\Gamma$ is uniformly bounded on each bounded $B \subset X$, i.e., $\{f(x): f \in \Gamma, x \in B\}$ is bounded. This is one of the foundation stones of functional analysis.

The uniform boundedness result has obtained many improvements [4] and $[7,8,9,10,11,12]$, and every pointwise bounded family of continuous linear operators from every separated ultrabarrelled (resp., barrelled) space to every topological vector (resp., locally convex) space is equicontinuous [14, p. 137, $140]$.

In this paper we would like to improve the equicontinuity theorem and the uniform boundedness principle by relaxing the linearity requirement forced on the mappings concerned. In fact, we shall find a meaningful extension of the family of linear operators and establish equicontinuity results for mappings in this extension.

Using the new equicontinuity theorem we shall extend the linear duality theory much wider in scope, and especially, we shall establish a new theory generalizing the usual distribution theory in forthcoming papers [5] and [3].

Also, our extended versions of the closed graph theorem [15] and the open mapping theorem [6] will play an important role in the demi-linear analysis.

\section{Motivations for demi-linearity}

For vector spaces $X, Y$ over the scalar field $\mathbb{K}$, every linear operator $T: X \rightarrow$ $Y$ has the exact splitting property:

Received October 11, 2007.

2000 Mathematics Subject Classification. Primary 46A30, 46F05.

Key words and phrases. demi-linear mappings, weakly demi-linear mappings, equicontinuity, uniform boundedness, demi-distributions, demi-linear duality. 


$$
T(x+t u)=T(x)+t T(u)
$$

for all $x, u \in X$ and $t \in \mathbb{K}$. If $X$ is not trivial and $\|\cdot\|: X \rightarrow \mathbb{R}$ is a norm and $\|\cdot\| \neq 0$, then $\|\cdot\|$ is not linear but $\|\cdot\|$ also has some splitting property: if $x, u \in X$ and $t \in \mathbb{K}$, then

$$
\|x+t u\|=\|x\|+s\|u\|
$$

for some $s \in[-|t|,|t|]$. Moreover, many nonlinear functions in $\mathbb{R}^{\mathbb{R}}$ have some local splitting property, e.g., if

$$
f \in\left\{\sin x, e^{x}-1, x / \sqrt{1+|x|}\right\},
$$

then for every $x \in \mathbb{R}$ and $u, t \in[-1,1]$, there exist $r, s \in \mathbb{R}$ such that $|r-1| \leq 10|t|,|s| \leq 10|t|$ and

$$
f(x+t u)=r f(x)+s f(u) .
$$

Definition 1.1. Let $C \geq 1$ and $\delta>0$. Then

(1) We denote by $\mathscr{L}_{C, \delta}(\mathbb{R}, \mathbb{R})$ the family of functions $f$ satisfying

(i) $f: \mathbb{R} \rightarrow \mathbb{R}$;

(ii) $f(0)=0$;

(iii) for all $x, u, t \in \mathbb{R},|u| \leq \delta,|t| \leq 1$ there exist $r, s \in \mathbb{R}$ satisfying $|r-1| \leq C|t|,|s| \leq C|t|$ and

$$
f(x+t u)=r f(x)+s f(u) .
$$

(2) We denote by $\mathscr{K}_{C, \delta}(\mathbb{R}, \mathbb{R})$ the family of functions $f$ satisfying

(i) $f: \mathbb{R} \rightarrow \mathbb{R}$;

(ii) $f(0)=0$;

(iii) for all $x, u, t \in \mathbb{R},|u| \leq \delta,|t| \leq 1$ there exists $s \in \mathbb{R}$ satisfying $|s| \leq C|t|$ and

$$
f(x+t u)=f(x)+s f(u) .
$$

It is trivial that

the family of linear functions $\subset \mathscr{K}_{C, \delta}(\mathbb{R}, \mathbb{R}) \subset \mathscr{L}_{C, \delta}(\mathbb{R}, \mathbb{R})$

for all $C \geq 1, \delta>0$.

Lemma 1.1. Every $f \in \mathscr{L}_{C, \delta}(\mathbb{R}, \mathbb{R})$ is continuous.

Proof. If $x_{n} \rightarrow x$ in $\mathbb{R}$, then we obtain for sufficiently large $n \in \mathbb{N}$ that $\mid x_{n}-$ $x \mid / \delta<1$ and

$$
f\left(x_{n}\right)=f\left(x+\frac{x_{n}-x}{\delta} \delta\right)=r_{n} f(x)+s_{n} f(\delta),
$$

where $\left|r_{n}-1\right| \leq C\left|x_{n}-x\right| / \delta$ and $\left|s_{n}\right| \leq C\left|x_{n}-x\right| / \delta$. Thus $f\left(x_{n}\right) \rightarrow f(x)$.

Lemma 1.2. If $f \in \mathscr{L}_{C, \delta}(\mathbb{R}, \mathbb{R}), f \neq 0$, then $f(u) \neq 0$ for every $0<|u| \leq \delta$. 
Proof. Suppose $0<|u| \leq \delta$ and $f(u)=0$. For nonzero $x \in \mathbb{R}$ we pick $n \in \mathbb{N}$ for which $|x /(n u)| \leq 1$, then

$$
\begin{aligned}
f(x) & =f\left(n \frac{x}{n u} u\right)=f\left[(n-1) \frac{x}{n u} u+\frac{x}{n u} u\right] \\
& =r_{1} f\left[(n-1) \frac{x}{n u} u\right]+s_{1} f(u)=r_{1} f\left[(n-2) \frac{x}{n u} u+\frac{x}{n u} u\right] \\
& =r_{1} r_{2} f\left[(n-2) \frac{x}{n u} u\right]=\cdots \\
& =r_{1} r_{2} \cdots r_{n-1} f\left(\frac{x}{n u} u\right)=r_{1} r_{2} \cdots r_{n-1} f\left(0+\frac{x}{n u} u\right) \\
& =r_{1} r_{2} \cdots r_{n-1} r_{n} f(0)+r_{1} r_{2} \cdots r_{n-1} s_{n} f(u)=0,
\end{aligned}
$$

where $\left|r_{i}-1\right| \leq C|x /(n u)|,\left|s_{i}\right| \leq C|x /(n u)|, i=1,2, \ldots, n$. Thus, $f(x)=0$ for every $x \in \mathbb{R}$.

Theorem 1.1. Let $f: \mathbb{R} \rightarrow \mathbb{R}$ be a function such that $f(0)=0$ and $f^{\prime}\left(x_{0}\right) \neq 0$ for some $x_{0} \in \mathbb{R}$. Also, let $\delta>0$. Then $f \in \mathscr{K}_{C, \delta}(\mathbb{R}, \mathbb{R})$ for some $C \geq 1$ if and only if

(1) $f$ is continuous,

(2) $f(u) \neq 0$ for every $0<|u| \leq \delta$,

(3) $\inf _{0<|u| \leq \delta}|f(u) / u|>0$,

(4) $\sup _{x, u \in \mathbb{R}, 0<|u| \leq \delta}|(f(x+u)-f(x)) / u|<+\infty$.

Proof. Suppose that $f \in \mathscr{K}_{C, \delta}(\mathbb{R}, \mathbb{R})$, where $C \geq 1$. By Lemmas 1.1 and 1.2, (1) and (2) hold for $f$. If $\inf _{0<|u| \leq \delta}|f(u) / u|=0$, then there exists a sequence $\left\{u_{n}\right\} \subset[-\delta, \delta] \backslash\{0\}$ such that $f\left(u_{n}\right) / u_{n} \rightarrow 0$. We may assume that $u_{n} \rightarrow u_{0} \in[-\delta, \delta]$. If $u_{0} \neq 0$, then $f\left(u_{n}\right) / u_{n} \rightarrow f\left(u_{0}\right) / u_{0} \neq 0$ by (1) and (2). So $u_{0}=0$ and $u_{n} \rightarrow 0$.

Since $f \in \mathscr{K}_{C, \delta}(\mathbb{R}, \mathbb{R})$ for all $n \in \mathbb{N} f\left(x_{0}+u_{n}\right)=f\left(x_{0}\right)+s_{n} f\left(u_{n}\right)$, where $\left|s_{n}\right| \leq C|1|=C$. It follows from (2) that

$$
\begin{aligned}
f\left(x_{0}+u_{n}\right) & =f\left(x_{0}\right)+f\left(x_{0}+u_{n}\right)-f\left(x_{0}\right) \\
& =f\left(x_{0}\right)+\frac{\left[f\left(x_{0}+u_{n}\right)-f\left(x_{0}\right)\right] / u_{n}}{f\left(u_{n}\right) / u_{n}} f\left(u_{n}\right),
\end{aligned}
$$

but

$$
\frac{f\left(x_{0}+u_{n}\right)-f\left(x_{0}\right)}{u_{n}} \rightarrow f^{\prime}\left(x_{0}\right) \neq 0
$$

and $f\left(u_{n}\right) / u_{n} \rightarrow 0$, so

$$
s_{n}=\frac{\left[f\left(x_{0}+u_{n}\right)-f\left(x_{0}\right)\right] / u_{n}}{f\left(u_{n}\right) / u_{n}} \rightarrow \infty .
$$

This is a contradiction and so (3) holds for $f$.

Let $x, u \in \mathbb{R}, 0<|u| \leq \delta$. Since $f(u)=f\left(\frac{u}{\delta} \delta\right)=s f(\delta)$, where $|s| \leq$ $C|u / \delta|=(C / \delta)|u|$ and $f(x+u)=f(x)+s_{1} f(u)$, where $\left|s_{1}\right| \leq C|1|=C$, 
$|f(u)| \leq(C / \delta)|f(\delta) u|$ and

$$
\left|\frac{f(x+u)-f(x)}{u}\right|=\left|\frac{s_{1} f(u)}{u}\right| \leq \frac{C^{2}}{\delta}|f(\delta)| .
$$

Thus, (4) holds for $f$.

Conversely, suppose (1), (2), (3), and (4) hold for $f$. Since $f(0)=0$ and $\inf _{0<|u| \leq \delta}|f(u) / u|=\inf _{0<|u| \leq \delta}|(f(0+u)-f(0)) / u|$,

$$
C=\left[\sup _{x, u \in \mathbb{R}, 0<|u| \leq \delta}\left|\frac{f(x+u)-f(x)}{u}\right| / \inf _{0<|u| \leq \delta}\left|\frac{f(u)}{u}\right|\right] \geq 1 .
$$

Then for $x, u, t \in \mathbb{R}, 0<|u| \leq \delta, 0<|t| \leq 1, f(u) \neq 0$ by (2) and

$$
f(x+t u)=f(x)+f(x+t u)-f(x)=f(x)+\left[\frac{f(x+t u)-f(x)}{t u} \frac{u}{f(u)} t\right] f(u),
$$

where

$$
\left|\frac{f(x+t u)-f(x)}{t u} \frac{u}{f(u)} t\right|=\left|\frac{f(x+t u)-f(x)}{t u} / \frac{f(u)}{u}\right||t| \leq C|t| .
$$

Thus, $f \in \mathscr{K}_{C, \delta}(\mathbb{R}, \mathbb{R})$.

Corollary 1.1. Let $f: \mathbb{R} \rightarrow \mathbb{R}$ be differentiable, $f^{\prime}(0) \neq 0$ and $\sup _{x \in \mathbb{R}}\left|f^{\prime}(x)\right|<$ $+\infty$. Then $f-f(0) \in \mathscr{K}_{C, \delta}(\mathbb{R}, \mathbb{R})$ for some $C \geq 1$ and $\delta>0$.

If $0<\sup _{x \in \mathbb{R}}|f(x)|<+\infty$, then $f: \mathbb{R} \rightarrow \mathbb{R}$ is nonlinear. For example, $\sin x$, $\arctan x, \tanh x$ and

$$
f(x)= \begin{cases}-\sqrt{2}, & x<-\sqrt{2} \\ x, & -\sqrt{2} \leq x \leq \sqrt{2} \\ \sqrt{2}, & x>\sqrt{2}\end{cases}
$$

etc.

Corollary 1.2. For every $C \geq 1$ and $\delta>0$, the set

$$
\left\{f \in \mathscr{K}_{C, \delta}(\mathbb{R}, \mathbb{R}): 0<\sup _{x \in \mathbb{R}}|f(x)|<+\infty\right\}
$$

is uncountable and so $\mathscr{K}_{C, \delta}(\mathbb{R}, \mathbb{R})$ includes uncountably many of nonlinear functions. In fact, for every $C \geq 1$ and $\delta>0$ the cardinal number

$$
\mid\left\{f \in \mathscr{K}_{C, \delta}(\mathbb{R}, \mathbb{R}): f \text { is nonlinear }\right\}|\geq| \mathbb{R}|=|\{f: \mathbb{R} \rightarrow \mathbb{R} \mid f \text { is linear }\} \mid .
$$

Corollary 1.3. For every $C_{0}>1$

$$
\mathscr{L}_{C_{0}, 1}(\mathbb{R}, \mathbb{R}) \backslash\left[\bigcup_{C \geq 1, \delta>0} \mathscr{K}_{C, \delta}(\mathbb{R}, \mathbb{R})\right] \neq \varnothing .
$$


Proof. Let $C_{0}>1$. Pick $\alpha \in(0,1)$ for which $e^{2 \alpha} \leq C_{0}$ and define $f: \mathbb{R} \rightarrow \mathbb{R}$ by $f(x)=e^{\alpha x}-1, x \in \mathbb{R}$. Since $\lim _{x \rightarrow+\infty} f^{\prime}(x)=\lim _{x \rightarrow+\infty} \alpha e^{\alpha x}=+\infty$, Theorem 1.1 shows that $f \notin \mathscr{K}_{C, \delta}(\mathbb{R}, \mathbb{R})$ for all $C \geq 1, \delta>0$. For $x, u, t \in$ $\mathbb{R}, 0<|u| \leq 1,0<|t| \leq 1$,

$$
\begin{aligned}
f(x+t u) & =e^{\alpha(x+t u)}-1=e^{\alpha(x+t u)}-e^{\alpha t u}+e^{\alpha t u}-1 \\
& =e^{\alpha t u}\left(e^{\alpha x}-1\right)+\frac{e^{\alpha t u}-1}{e^{\alpha u}-1}\left(e^{\alpha u}-1\right)=e^{\alpha t u} f(x)+\frac{e^{\alpha t u}-1}{e^{\alpha u}-1} f(u)
\end{aligned}
$$

and

$$
\begin{gathered}
\left|e^{\alpha t u}-1\right|=\left|e^{\theta \alpha t u} \alpha t u\right| \leq e^{\alpha}|t|<C_{0}|t|, \\
\left|\frac{e^{\alpha t u}-1}{e^{\alpha u}-1}\right|=\left|\frac{e^{\theta \alpha t u} \alpha t u}{e^{\eta \alpha u} \alpha u}\right|=e^{(\theta t-\eta) \alpha u}|t| \leq e^{2 \alpha}|t| \leq C_{0}|t|,
\end{gathered}
$$

where $\theta, \eta \in(0,1)$. Thus, $f \in \mathscr{L}_{C_{0}, 1}(\mathbb{R}, \mathbb{R})$.

\section{Demi-linear and weakly demi-linear mappings}

Let $X$ be a topological vector space and $\mathcal{N}(X)$ the family of neighborhoods of $0 \in X$. Then we denote by $C(0)$ the set of complex valued functions $\gamma$ satisfying

(1) $\gamma: \mathbb{C} \rightarrow \mathbb{C}$

(2) $\lim _{t \rightarrow 0} \gamma(t)=\gamma(0)=0$;

(3) $|\gamma(t)| \geq|t|$ if $|t| \leq 1$.

Definition 2.1. A mapping $f: X \rightarrow Y$ is said to be demi-linear if $f(0)=0$ and there exist $\gamma \in C(0)$ and $U \in \mathcal{N}(X)$ such that every $x \in X, u \in U$ and $t \in\{t \in \mathbb{K}:|t| \leq 1\}$ yield $r, s \in \mathbb{K}$ for which $|r-1| \leq|\gamma(t)|,|s| \leq|\gamma(t)|$ and $f(x+t u)=r f(x)+s f(u)$.

We denote by $\mathscr{L}_{\gamma, U}(X, Y)$ the demi-linear mappings related to $\gamma \in C(0)$ and $U \in \mathcal{N}(X)$, and by $\mathscr{K}_{\gamma, U}(X, Y)$ the subfamily of $\mathscr{L}_{\gamma, U}(X, Y)$ satisfying the following property: if $x \in X, u \in U$ and $|t| \leq 1$, then

$$
f(x+t u)=f(x)+s f(u)
$$

for some $s$ with $|s| \leq|\gamma(t)|$.

If $\gamma(t)=C t$ with $C \geq 1$, then we write that $\mathscr{L}_{\gamma, U}(X, Y)=\mathscr{L}_{C, U}(X, Y)$ and $\mathscr{K}_{\gamma, U}(X, Y)=\mathscr{K}_{C, U}(X, Y)$. Moreover, if $X$ is normed and

$$
U=B_{\delta}=\{x \in X:\|x\| \leq \delta\},
$$

then $\mathscr{L}_{\gamma, \delta}(X, Y)=\mathscr{L}_{\gamma, U}(X, Y)$ and $\mathscr{K}_{\gamma, \delta}(X, Y)=\mathscr{K}_{\gamma, U}(X, Y)$. Thus, both $\mathscr{L}_{C, \delta}(\mathbb{R}, \mathbb{R})$ and $\mathscr{K}_{C, \delta}(\mathbb{R}, \mathbb{R})$ are families of demi-linear functions in $\mathbb{R}^{\mathbb{R}}$.

Theorem 2.1. Let $X$ be a nontrivial normed space and $Y$ a nontrivial vector space. For every $C>1, \delta>0$ and $U=\{u \in X:\|u\| \leq \delta\}$ the family of nonlinear mappings in $\mathscr{L}_{C, \delta}(X, Y)$ is uncountable. Especially, every nonzero linear operator $T: X \rightarrow Y$ produces uncountably many of nonlinear mappings in $\mathscr{L}_{C, \delta}(X, Y)$. 
Proof. Pick $\eta \in \mathscr{K}_{C, \delta}(\mathbb{R}, \mathbb{R})$ for which $0<K_{0}=\sup _{t \in \mathbb{R}}|\eta(t)|<+\infty$. Since $\lim _{t \rightarrow+\infty} \frac{t+(1+C) K_{0}}{t-K_{0}}=1<C$, there exists $K_{1}>2 K_{0}$ such that

$$
\frac{K_{0}}{K-K_{0}}<1, \quad \frac{K+(1+C) K_{0}}{K-K_{0}}<C
$$

for every $K \geq K_{1}$. For a nonzero linear operator $T: X \rightarrow Y$ and $K \geq K_{1}$ define $f_{T, K}: X \rightarrow Y$ by

$$
f_{T, K}(x)=[K+\eta(\|x\|)] T(x), x \in X .
$$

Let $x, u \in X, 0<\|u\| \leq \delta$ and $0<|t| \leq 1$. Since $\|x+t u\|=\|x\|+s\|u\|$ for some $s \in[-|t|,|t|]$ and $\eta \in \mathscr{K}_{C, \delta}(\mathbb{R}, \mathbb{R})$, there exists $\alpha$ with $|\alpha| \leq C|s| \leq C|t|$ such that

$$
\begin{aligned}
& f_{T, K}(x+t u) \\
= & {[K+\eta(\|x+t u\|)] T(x+t u) } \\
= & {[K+\eta(\|x\|+s\|u\|)] T(x+t u) } \\
= & {[K+\eta(\|x\|)+\alpha \eta(\|u\|)][T(x)+t T(u)] } \\
= & \frac{K+\eta(\|x\|)+\alpha \eta(\|u\|)}{K+\eta(\|x\|)} f_{T, K}(x)+\frac{K+\eta(\|x\|)+\alpha \eta(\|u\|)}{K+\eta(\|u\|)} t f_{T, K}(u),
\end{aligned}
$$

where

$$
\begin{gathered}
\left|\frac{K+\eta(\|x\|)+\alpha \eta(\|u\|)}{K+\eta(\|x\|)}-1\right|=\frac{|\alpha \eta(\|u\|)|}{K+\eta(\|x\|)} \leq \frac{K_{0} C|t|}{K-K_{0}}<C|t|, \\
\left|\frac{K+\eta(\|x\|)+\alpha \eta(\|u\|)}{K+\eta(\|u\|)} t\right| \leq \frac{K+K_{0}+C|t| K_{0}}{K-K_{0}}|t| \leq \frac{K+(1+C) K_{0}}{K-K_{0}}|t|<C|t| .
\end{gathered}
$$

Thus, $f_{T, K} \in \mathscr{L}_{C, \delta}(X, Y)$.

By Corollary $1.2, \mathscr{K}_{C, \delta}(\mathbb{R}, \mathbb{R})$ includes uncountably many of nonlinear mappings which can be used to construct demi-linear mappings as above $f_{T, K}$.

Definition 2.2. Let $X$ be a topological vector space and $Y$ a locally convex space with dual $Y^{\prime}$. A mapping $f: X \rightarrow Y$ is said to be weakly demi-linear if $f(0)=0$ and there exist $\gamma \in C(0)$ and $U \in \mathcal{N}(X)$ such that $y^{\prime} \circ f \in \mathscr{L}_{\gamma, U}(X, \mathbb{C})$ for each $y^{\prime} \in Y^{\prime}$.

Let $\mathscr{W}_{\gamma, U}(X, Y)=\left\{f \in Y^{X}: f(0)=0\right.$ and $y^{\prime} \circ f \in \mathscr{L}_{\gamma, U}(X, \mathbb{C})$ for all $\left.y^{\prime} \in Y^{\prime}\right\}$. We write $\mathscr{W}_{\gamma, U}(X, Y)=\mathscr{W}_{C, U}(X, Y)$ when $\gamma(t)=C t$. Obviously, $\mathscr{L}_{\gamma, U}(X, Y) \subset \mathscr{W}_{\gamma, U}(X, Y)$ but $\mathscr{L}_{\gamma, U}(X, Y)$ can be a proper subfamily of $\mathscr{W}_{\gamma, U}(X, Y)$.

Example 2.1. For $(a, b) \in \mathbb{R}^{2}$ let $\|(a, b)\|=|2 a+b|$. Then $\|\cdot\|$ is a seminorm on $\mathbb{R}^{2}$ and the seminormed space $\left(\mathbb{R}^{2},\|\cdot\|\right)$ is locally convex. Let $\xi:\left(\mathbb{R}^{2},\|\cdot\|\right) \rightarrow \mathbb{R}$ be a continuous linear functional. Then $\xi=(\alpha, \beta) \in \mathbb{R}^{2}$, 
$\xi(a, b)=\langle(\alpha, \beta),(a, b)\rangle=\alpha a+\beta b$ for all $(a, b) \in \mathbb{R}^{2}$. Since

$$
\begin{aligned}
\left\|\left(n+\frac{1}{n},-2 n\right)\right\| & =\left|2 n+\frac{2}{n}-2 n\right|=\frac{2}{n} \rightarrow 0, \\
\xi\left(n+\frac{1}{n},-2 n\right) & =\left\langle(\alpha, \beta),\left(n+\frac{1}{n},-2 n\right)\right\rangle \\
& =\alpha n+\frac{\alpha}{n}-2 \beta n=(\alpha-2 \beta) n+\frac{\alpha}{n} \rightarrow 0
\end{aligned}
$$

and so $\alpha=2 \beta,(\alpha, \beta)=(2 \beta, \beta)$.

Conversely, if $\left\|\left(a_{n}, b_{n}\right)-(a, b)\right\| \rightarrow 0$, then $2 a_{n}+b_{n} \rightarrow 2 a+b$ and, for every $\beta \in \mathbb{R}$,

$\left\langle(2 \beta, \beta),\left(a_{n}, b_{n}\right)\right\rangle=2 \beta a_{n}+\beta b_{n}=\beta\left(2 a_{n}+b_{n}\right) \rightarrow \beta(2 a+b)=\langle(2 \beta, \beta),(a, b)\rangle$, so $(2 \beta, \beta) \in\left(\mathbb{R}^{2},\|\cdot\|\right)^{\prime}$ for all $\beta \in \mathbb{R}$, i.e., $(\mathbb{R},\|\cdot\|)^{\prime}=\{(2 \beta, \beta): \beta \in \mathbb{R}\}$.

Let

$$
\eta(x)= \begin{cases}1, & x>1 \\ x, & -1 \leq x \leq 1 \\ -1, & x<-1\end{cases}
$$

and define $f: \mathbb{R} \rightarrow \mathbb{R}^{2}$ by $f(x)=(x, \eta(x))$ for all $x \in \mathbb{R}$. For $0<\delta<1$ pick $u \in(0, \delta)$ and $x, t \in(0,1)$ for which $x+t u>1$, e.g., $x=1-\delta / 5, u=\delta / 2, t=$ $1 / 2$. Then $\eta(x+t u)=1$ and $f(x+t u)=(x+t u, 1)$. If there exist $r, s \in \mathbb{R}$ such that $f(x+t u)=r f(x)+s f(u)$, i.e.,

$$
(x+t u, 1)=r(x, x)+s(u, u)=(r x+s u, r x+s u),
$$

then $r x+s u=x+t u>1=r x+s u$. This contradiction shows that $f: \mathbb{R} \rightarrow \mathbb{R}^{2}$ is not demi-linear: $f \notin \mathscr{L}_{C,[-\delta, \delta]}\left(\mathbb{R}, \mathbb{R}^{2}\right)$ for all $C \geq 1, \delta>0$.

However, $f \in \mathscr{W}_{1,[-1,1]}\left(\mathbb{R},\left(\mathbb{R}^{2},\|\cdot\|\right)\right)$. To see this, let $(2 \beta, \beta) \in\left(\mathbb{R}^{2}\right.$, $\|\cdot\|)^{\prime}, \beta \neq 0$. By Theorem 1.1, $\eta \in \mathscr{K}_{1,[-1,1]}(\mathbb{R}, \mathbb{R})$ and so for every $x \in \mathbb{R}$ and $t, u \in[-1,1] \backslash\{0\}$ there exists $s \in[-|t|,|t|]$ such that $\eta(x+t u)=\eta(x)+s \eta(u)=$ $\eta(x)+s u$ and

$$
\begin{aligned}
\langle(2 \beta, \beta), f(x+t u)\rangle & =\langle(2 \beta, \beta),(x+t u, \eta(x)+s u)\rangle \\
& =\langle(2 \beta, \beta),(x, \eta(x))\rangle+\langle(2 \beta, \beta),(t u, s u)\rangle \\
& =\langle(2 \beta, \beta), f(x)\rangle+2 \beta t u+\beta s u \\
& =\langle(2 \beta, \beta), f(x)\rangle+\frac{2 \beta t u+\beta s u}{2 \beta u+\beta u}\langle(2 \beta, \beta),(u, u)\rangle \\
& =\langle(2 \beta, \beta), f(x)\rangle+\frac{2 t+s}{3}\langle(2 \beta, \beta), f(u)\rangle,
\end{aligned}
$$

where $|2 t+s| / 3 \leq(2|t|+|s|) / 3 \leq|t|$. Thus, $\langle(2 \beta, \beta), f(\cdot)\rangle \in \mathscr{L}_{1,[-1,1]}(\mathbb{R}, \mathbb{R})$ and so $f \in \mathscr{W}_{1,[-1,1]}\left(\mathbb{R},\left(\mathbb{R}^{2},\|\cdot\|\right)\right)$. 


\section{Equicontinuity}

For topological vector spaces $X, Y$, a family $\Gamma \subset Y^{X}$ is said to be equicontinuous at $x \in X$ if for every $V \in \mathcal{N}(Y)$ there exists $U \in \mathcal{N}(X)$ such that $f(x+U) \subset f(x)+V$ for all $f \in \Gamma$. If $\Gamma$ is equicontinuous at each $x \in X$, then $\Gamma$ is said to be equicontinuous on $X$. We refer to [1] and [13, p. 129] for standard definitions and results.

Henceforth, $X$ and $Y$ are topological vector spaces. In the notations $\mathscr{L}_{\gamma, U}(X$, $Y$ ) and $\mathscr{W}_{\gamma, U}(X, Y), \gamma \in C(0)$ and $U \in \mathcal{N}(X)$ is both closed and balanced. $\Gamma \subset Y^{X}$ is said to be pointwise bounded if $\{f(x): f \in \Gamma\}$ is bounded at each $x \in X$.

Theorem 3.1. If $X$ is of second category and $\Gamma \subset \mathscr{L}_{\gamma, U}(X, Y)$ is a pointwise bounded family of continuous mappings, then $\Gamma$ is equicontinuous on $X$.

Proof. Let $V \in \mathcal{N}(Y)$. Pick a closed balanced set $W \in \mathcal{N}(Y)$ satisfying $W+$ $W \subset V$, and let

$$
W_{0}=\frac{1}{1+|\gamma(-1)|} W, \quad M=U \cap\left(\bigcap_{f \in \Gamma} f^{-1}\left(W_{0}\right)\right) .
$$

Since each $f \in \Gamma$ is continuous and both $U$ and $W_{0}$ are closed, $M$ is closed and $0 \in M$.

Let $x \in X$. Pick $\alpha \in(0,1)$ so that $\alpha x \in U$. Since $\{f(\alpha x): f \in \Gamma\}$ is bounded, there exists $\varepsilon \in(0,1)$ such that $s f(\alpha x) \in W_{0}$ for all $f \in \Gamma,|s| \leq \varepsilon$. Observing $\lim _{t \rightarrow 0} \gamma(t)=0$, pick $\delta \in(0,1)$ such that $|\gamma(t)|<\varepsilon$ when $|t| \leq \delta$. If $f \in \Gamma$ and $|t| \leq \delta$, then $f(t \alpha x)=s f(\alpha x)$ with $|s| \leq|\gamma(t)|<\varepsilon$ and so $f(t \alpha x) \in W_{0}$. Thus, $t \alpha x \in \bigcap_{f \in \Gamma} f^{-1}\left(W_{0}\right)$ for every $|t| \leq \delta$. Since $U$ is balanced, it follows that $t \alpha x \in U$ when $|t| \leq \delta$ and

$$
t \alpha x \in U \cap\left(\bigcap_{f \in \Gamma} f^{-1}\left(W_{0}\right)\right)=M
$$

for every $|t| \leq \delta$. Then for $1 / n<\alpha \delta,(1 / n) x=(1 / \alpha n) \alpha x \in M$, so $x \in n M$. Thus, $X=\bigcup_{n=1}^{\infty} n M$ and $M$ has nonempty interior by the Baire category theorem. Hence,

$$
M-M=\{x-z: x, z \in M\} \in \mathcal{N}(X) .
$$

Let $x, z \in M$ and $f \in \Gamma$. Then

$$
f(x), f(z) \in W_{0}=\frac{1}{1+|\gamma(-1)|} W
$$

and so

$$
f(x)=\frac{1}{1+|\gamma(-1)|} y_{1}, \quad f(z)=\frac{1}{1+|\gamma(-1)|} y_{2},
$$

where $y_{1}, y_{2} \in W$. Since $z \in M \subset U, f(x-z)=r f(x)+s f(z)$, where $|r-1| \leq|\gamma(-1)|,|s| \leq|\gamma(-1)|$. But $W$ is balanced and $|r| \leq 1+|\gamma(-1)|$, it 
follows that

$$
f(x-z)=r f(x)+s f(z)=\frac{r}{1+|\gamma(-1)|} y_{1}+\frac{s}{1+|\gamma(-1)|} y_{2} \in W+W \subset V .
$$

Thus, $M-M \in \mathcal{N}(X)$ and $f(M-M) \subset V$ for all $f \in \Gamma$, i.e., $\Gamma$ is equicontinuous at $0 \in X$.

Let $x \in X$ and $V \in \mathcal{N}(Y)$. Pick a balanced set $W \in \mathcal{N}(Y)$ so that $W+W \subset$ $V$. Since $\Gamma$ is equicontinuous at $0 \in X$, there exists $U_{0} \in \mathcal{N}(X)$ satisfying $f\left(U_{0}\right) \subset W$ for all $f \in \Gamma$. But $\{f(x): f \in \Gamma\}$ is bounded so there exists $\varepsilon \in(0,1)$ for which $t f(x) \in W$ for every $f \in \Gamma,|t| \leq \varepsilon$. Pick $\delta \in(0,1)$ so that $|\gamma(t)|<\varepsilon$ when $|t| \leq \delta$. Then for $f \in \Gamma$ and $z=(\delta / 2) z_{0} \in(\delta / 2)\left(U_{0} \cap U\right)$ we have

$$
f(x+z)=f\left(x+\frac{\delta}{2} z_{0}\right)=r f(x)+s f\left(z_{0}\right)=f(x)+(r-1) f(x)+s f\left(z_{0}\right),
$$

where $|r-1| \leq\left|\gamma\left(\frac{\delta}{2}\right)\right|<\varepsilon$ and $|s| \leq\left|\gamma\left(\frac{\delta}{2}\right)\right|<\varepsilon<1$. Therefore, it follows that

$$
(r-1) f(x) \in W, \quad s f\left(z_{0}\right) \in s f\left(U_{0}\right) \subset s W \subset W
$$

and

$$
f(x+z)=f(x)+(r-1) f(x)+s f\left(z_{0}\right) \in f(x)+W+W \subset f(x)+V .
$$

Thus we obtain

$$
\frac{\delta}{2}\left(U_{0} \cap U\right) \in \mathcal{N}(X), \quad f\left[x+\frac{\delta}{2}\left(U_{0} \cap U\right)\right] \subset f(x)+V
$$

for all $f \in \Gamma$. This shows that $\Gamma$ is equicontinuous at each $x \in X$.

Corollary 3.1. Suppose that $X$ is of second category and $\Gamma \subset \mathscr{L}_{\gamma, U}(X, Y)$ is a pointwise bounded family of continuous mappings. If $\left(x_{\alpha}\right)_{\alpha \in I}$ is a net in $X$ such that $x_{\alpha} \rightarrow x$, then $\lim _{\alpha} f\left(x_{\alpha}\right)=f(x)$ uniformly for $f \in \Gamma$.

Proof. Let $V \in \mathcal{N}(Y)$. Then, by Theorem 3.1, there exists $W \in \mathcal{N}(X)$ for which $f(x+W) \subset f(x)+V$ for every $f \in \Gamma$. Since $x_{\alpha} \rightarrow x$, there exists $\alpha_{0} \in I$ such that $x_{\alpha}-x \in W$ for all $\alpha \geq \alpha_{0}$. Then

$$
f\left(x_{\alpha}\right)-f(x)=f\left(x+x_{\alpha}-x\right)-f(x) \in f(x+W)-f(x) \subset V
$$

for all $\alpha \geq \alpha_{0}, f \in \Gamma$.

Theorem 3.2. If $X$ is of second category and $\left\{f_{n}\right\} \subset \mathscr{L}_{\gamma, U}(X, Y)$ is a sequence of continuous mappings such that $\lim _{n} f_{n}(x)=f(x)$ exists at each $x \in X$, then $f \in \mathscr{L}_{\gamma, U}(X, Y)$ and $f$ is also continuous.

Proof. Let $x \in X, u \in U$ and $|t| \leq 1$. Then

$$
f(x+t u)=\lim _{n} f_{n}(x+t u)=\lim _{n}\left[r_{n} f_{n}(x)+s_{n} f_{n}(u)\right],
$$


where $\left|r_{n}-1\right| \leq|\gamma(t)|$ and $\left|s_{n}\right| \leq|\gamma(t)|$. There exists an integer sequence $n_{1}<n_{2}<\cdots$ such that $r_{n_{k}} \rightarrow r, s_{n_{k}} \rightarrow s$ and

$$
|r-1|=\lim _{k}\left|r_{n_{k}}-1\right| \leq|\gamma(t)|, \quad|s|=\lim _{k}\left|s_{n_{k}}\right| \leq|\gamma(t)| .
$$

Moreover,

$$
f(x+t u)=\lim _{k} f_{n_{k}}(x+t u)=\lim _{k}\left[r_{n_{k}} f_{n_{k}}(x)+s_{n_{k}} f_{n_{k}}(u)\right]=r f(x)+s f(u) .
$$

Thus, $f \in \mathscr{L}_{\gamma, U}(X, Y)$.

Since $\lim _{n} f_{n}(x)=f(x)$ exists at each $x \in X,\left\{f_{n}: n \in \mathbb{N}\right\}$ is pointwise bounded. If $x_{\alpha} \rightarrow x$ in $X$, then Corollary 3.1 shows that

$$
\lim _{\alpha} f\left(x_{\alpha}\right)=\lim _{\alpha} \lim _{n} f_{n}\left(x_{\alpha}\right)=\lim _{n} \lim _{\alpha} f_{n}\left(x_{\alpha}\right)=\lim _{n} f_{n}(x)=f(x) .
$$

Thus, $f$ is continuous.

The most important consequence of Theorem 3.1 is the following substantial improvement of the classical uniform boundedness principle.

Theorem 3.3. If $X$ is of second category and $\Gamma \subset \mathscr{L}_{\gamma, U}(X, Y)$ is a pointwise bounded family of continuous mappings, then $\Gamma$ is uniformly bounded on each bounded subset of $X$, i.e., $\{f(x): f \in \Gamma, x \in B\}$ is bounded for every bounded $B \subset X$.

Proof. Suppose that $B \subset X$ is bounded and $V \in \mathcal{N}(Y)$ is balanced. By Theorem 3.1, there is a balanced set $U_{0} \in \mathcal{N}(X)$ such that $U_{0} \subset U$ and $f\left(U_{0}\right) \subset V$ for all $f \in \Gamma$. Pick $n_{0} \in \mathbb{N}$ for which $\frac{1}{n_{0}} B \subset U_{0}$. Observe that $V$ decides $U_{0}$, both $U_{0}$ and $B$ decide $n_{0}$ so $n_{0}$ is independent of every individual $x \in B$.

If $x \in B$ and $f \in \Gamma$, then $x=n_{0} \frac{x}{n_{0}}, \frac{x}{n_{0}} \in U_{0} \subset U$ and so

$$
\begin{aligned}
f(x)= & f\left(n_{0} \frac{x}{n_{0}}\right) \\
= & f\left[\left(n_{0}-1\right) \frac{x}{n_{0}}+\frac{x}{n_{0}}\right] \\
= & r_{1} f\left[\left(n_{0}-1\right) \frac{x}{n_{0}}\right]+s_{1} f\left(\frac{x}{n_{0}}\right) \\
= & r_{1} r_{2} f\left[\left(n_{0}-2\right) \frac{x}{n_{0}}\right]+r_{1} s_{2} f\left(\frac{x}{n_{0}}\right)+s_{1} f\left(\frac{x}{n_{0}}\right) \\
& \quad \cdots \quad \ldots \\
= & \left(r_{1} \cdots r_{n_{0}-1}+r_{1} \cdots r_{n_{0}-2} s_{n_{0}-1}+\cdots+r_{1} s_{2}+s_{1}\right) f\left(\frac{x}{n_{0}}\right),
\end{aligned}
$$

where $\left|r_{i}-1\right| \leq|\gamma(1)|,\left|s_{i}\right| \leq|\gamma(1)|, i=1,2, \ldots, n_{0}-1$. Let $\alpha_{x}=r_{1} \cdots r_{n_{0}-1}+$ $r_{1} \cdots r_{n_{0}-2} s_{n_{0}-1}+\cdots+r_{1} s_{2}+s_{1}$. Then $\left|\alpha_{x}\right| \leq n_{0}(1+|\gamma(1)|)^{n_{0}-1}$ and

$$
t f(x)=t \alpha_{x} f\left(\frac{x}{n_{0}}\right) \in t \alpha_{x} f\left(U_{0}\right) \subset t \alpha_{x} V \subset V
$$


for every $|t| \leq 1 /\left[n_{0}(1+|\gamma(1)|)^{n_{0}-1}\right]$. Thus,

$$
t\{f(x): f \in \Gamma, x \in B\} \subset V
$$

for every $|t| \leq 1 /\left[n_{0}(1+|\gamma(1)|)^{n_{0}-1}\right]$.

A mapping $f: X \rightarrow Y$ is said to be bounded if $f(B)$ is bounded for each bounded $B \subset X$. Even bounded linear functionals on a locally convex space need not be continuous. However, if $X$ is metrizable, then a linear operator $f: X \rightarrow Y$ is continuous if and only if $f$ is bounded.

Lemma 3.1. Let $f \in \mathscr{L}_{\gamma, U}(X, Y)$ is continuous. Then $f$ is bounded.

Proof. Suppose that $B \subset X$ is bounded and $V \in \mathcal{N}(Y)$ is balanced. Pick a balanced set $U_{0} \in \mathcal{N}(X)$ such that $U_{0} \subset U$ and $f\left(U_{0}\right) \subset V$. As in the proof of Theorem 3.3, $\frac{1}{n_{0}} B \subset U_{0}$ for some $n_{0} \in \mathbb{N}$ and

$$
t f(B) \subset V \quad \text { for every }|t| \leq 1 /\left[n_{0}(1+|\gamma(1)|)^{n_{0}-1}\right] .
$$

Lemma 3.2. Let $X$ be metrizable and $f \in \mathscr{L}_{\gamma, U}(X, Y)$. Then $f$ is continuous if and only if $f$ is bounded.

Proof. Suppose that $f$ is bounded and $x_{n} \rightarrow x$ in $X$. Since $X$ is metrizable and $x_{n}-x \rightarrow 0$, there exist $\left\{u_{n}\right\} \subset X$ and $\left\{t_{n}\right\} \subset \mathbb{C}$ such that $u_{n} \rightarrow 0, t_{n} \rightarrow 0$ and $\left\{x_{n}-x\right\}=\left\{t_{n} u_{n}\right\}\left[2\right.$, p. 380]. Pick $n_{0} \in \mathbb{N}$ for which $u_{n} \in U$ and $\left|t_{n}\right|<1$ whenever $n>n_{0}$.

Since $\left\{u_{n}\right\}$ is bounded and

$$
\begin{aligned}
f\left(x_{n}\right)-f(x) & =f\left(x+x_{n}-x\right)-f(x)=f\left(x+t_{n} u_{n}\right)-f(x) \\
& =r_{n} f(x)+s_{n} f\left(u_{n}\right)-f(x)=\left(r_{n}-1\right) f(x)+s_{n} f\left(u_{n}\right),
\end{aligned}
$$

where $\left|r_{n}-1\right| \leq\left|\gamma\left(t_{n}\right)\right|$ and $\left|s_{n}\right| \leq\left|\gamma\left(t_{n}\right)\right|$ for all $n>n_{0},\left\{f\left(u_{n}\right)\right\}$ is bounded and $r_{n} \rightarrow 1, s_{n} \rightarrow 0$ so $f\left(x_{n}\right) \rightarrow f(x)$.

Now Theorem 3.3 gives a substantial improvement of the classical resonance theorem as follows.

Theorem 3.4. Suppose $X$ is of second category and metrizable. If $\Gamma \subset$ $\mathscr{L}_{\gamma, U}(X, Y)$ is a pointwise bounded family of bounded mappings, then $\Gamma$ is uniformly bounded on each bounded set in $X$.

\section{Further improvements}

Let $Y$ be a locally convex space with the dual $Y^{\prime}$. If $f \in \mathscr{W}_{\gamma, U}(X, Y)$, i.e., $f(0)=0$ and $y^{\prime} \circ f \in \mathscr{L}_{\gamma, U}(X, \mathbb{C})$ for each $y^{\prime} \in Y^{\prime}$, then for every $x \in X, u \in$ $U,|t| \leq 1$ and $y^{\prime} \in Y^{\prime}$ there exist scalars $r_{y^{\prime}}$ and $s_{y^{\prime}}$ such that

$$
\left|r_{y^{\prime}}-1\right| \leq|\gamma(t)|, \quad\left|s_{y^{\prime}}\right| \leq|\gamma(t)|
$$

and

$$
y^{\prime}(f(x+t u))=r_{y^{\prime}} y^{\prime}(f(x))+s_{y^{\prime}} y^{\prime}(f(u)) .
$$


If $f: X \rightarrow Y$ is continuous, then $y^{\prime} \circ f: X \rightarrow \mathbb{C}$ is continuous for each $y^{\prime} \in Y^{\prime}$. The converse is not true, e.g., if $f:\left(c_{0}\right.$, weak $) \rightarrow\left(c_{0},\|\cdot\|_{\infty}\right), f(x)=x$ for $x \in c_{0}$, then $x^{\prime} \circ f:\left(c_{0}\right.$, weak $) \rightarrow \mathbb{C}$ is continuous for each $x^{\prime} \in c_{0}^{\prime}$ but $f:\left(c_{0}\right.$, weak $) \rightarrow\left(c_{0},\|\cdot\|_{\infty}\right)$ is not continuous.

Recall that $\mathscr{L}_{\gamma, U}(X, Y) \varsubsetneqq \mathscr{W}_{\gamma, U}(X, Y)$, in general (see Example 2.1).

Theorem 4.1. Suppose that $X$ is of second category and $Y$ is a locally convex space with the dual $Y^{\prime}$. If $\Gamma \subset \mathscr{W}_{\gamma, U}(X, Y)$ is pointwise bounded and $y^{\prime} \circ f$ : $X \rightarrow \mathbb{C}$ is continuous whenever $y^{\prime} \in Y^{\prime}$ and $f \in \Gamma$, then $\Gamma$ is equicontinuous on $X$ and, in particular, each $f \in \Gamma$ is continuous.

Proof. For every $W \in \mathcal{N}(Y)$ there is a barrel $V \in \mathcal{N}(Y)$ for which $V \subset W[14$, p. 92]. Moreover, for each $W \in \mathcal{N}(Y)$ the polar

$$
W^{\circ}=\left\{y^{\prime} \in Y^{\prime}:\left|y^{\prime}(y)\right| \leq 1 \quad \text { for all } y \in W\right\}
$$

is equicontinuous on $Y$ [14, p. 129].

Let $W \in \mathcal{N}(Y)$. Pick a barrel $V \in \mathcal{N}(Y)$ for which $V \subset W$. If $x \in X$ for which $\sup _{f \in \Gamma, y^{\prime} \in V^{\circ}}\left|y^{\prime}(f(x))\right|=+\infty$, then there exist $\left\{f_{n}\right\} \subset \Gamma$ and $\left\{y_{n}^{\prime}\right\} \subset$ $V^{\circ}$ such that $\left|y_{n}^{\prime}\left(f_{n}(x)\right)\right|>n$ and so $\left|y_{n}^{\prime}\left(\frac{1}{n} f_{n}(x)\right)\right|>1$ for all $n \in \mathbb{N}$. But $\{f(x): f \in \Gamma\}$ is bounded so $\frac{1}{n} f_{n}(x) \rightarrow 0$, and since $V^{\circ}$ is equicontinuous on $Y$, it follows that $\lim _{n} y^{\prime}\left(\frac{1}{n} f_{n}(x)\right)=0$ uniformly for $y^{\prime} \in V^{\circ}$. This is a contradiction and so $\left\{y^{\prime}(f(x)): y^{\prime} \in V^{\circ}, f \in \Gamma\right\}$ is bounded at each $x \in X$. Since

$$
\left\{y^{\prime} \circ f: y^{\prime} \in V^{\circ}, f \in \Gamma\right\} \subset \mathscr{L}_{\gamma, U}(X, \mathbb{C})
$$

and each $y^{\prime} \circ f$ is continuous by the hypothesis, Theorem 3.1 shows that $\left\{y^{\prime} \circ f\right.$ : $\left.y^{\prime} \in V^{\circ}, f \in \Gamma\right\}$ is equicontinuous on $X$.

Let $x_{0} \in X$. There exists $U_{0} \in \mathcal{N}(X)$ such that

$$
\left|y^{\prime}\left[f\left(x_{0}+u\right)-f\left(x_{0}\right)\right]\right|=\left|y^{\prime}\left(f\left(x_{0}+u\right)\right)-y^{\prime}\left(f\left(x_{0}\right)\right)\right|<1
$$

for every $y^{\prime} \in V^{\circ}, f \in \Gamma$ and $u \in U_{0}$. But, $V$ is a barrel, so $V^{\circ \circ}=V$ by the bipolar theorem $[14$, p. 112]. Therefore, we have

$$
f\left(x_{0}+u\right)-f\left(x_{0}\right) \in V^{\circ \circ}=V \subset W
$$

for every $f \in \Gamma, u \in U_{0}$. This shows that $\Gamma$ is equicontinuous at every $x_{0} \in$ $X$.

Corollary 4.1. Suppose that $X$ is of second category and $Y$ is a locally convex space with the dual $Y^{\prime}$. Then $f \in \mathscr{W}_{\gamma, U}(X, Y)$ is continuous if and only if $y^{\prime} \circ f: X \rightarrow \mathbb{C}$ is continuous for each $y^{\prime} \in Y^{\prime}$.

Proof. $\Gamma=\{f\}$ is pointwise bounded. Theorem 4.1.

Theorem 4.2. Suppose that $X$ is of second category and $Y$ is a locally convex space with the dual $Y^{\prime}$. If $\Gamma \subset \mathscr{W}_{\gamma, U}(X, Y)$ is pointwise bounded and $y^{\prime} \circ f$ : $X \rightarrow \mathbb{C}$ is continuous whenever $y^{\prime} \in Y^{\prime}$ and $f \in \Gamma$, then $\Gamma$ is uniformly bounded on each bounded subset of $X$. 
Proof. Let $y^{\prime} \in Y^{\prime}$. Then $\left\{y^{\prime} \circ f: f \in \Gamma\right\} \subset \mathscr{L}_{\gamma, U}(X, \mathbb{C})$ and $\left\{y^{\prime}(f(x)): f \in \Gamma\right\}$ is bounded at each $x \in X$. If $B \subset X$ is bounded, then $\left\{y^{\prime}(f(x)): f \in \Gamma, x \in\right.$ $B\}$ is bounded by Theorem 3.3. Since $y^{\prime} \in Y^{\prime}$ is arbitrary, it follows that $\{f(x): f \in \Gamma, x \in B\}$ is bounded in (Y,weak). Therefore, by the Mackey theorem [14, p. 114], $\{f(x): f \in \Gamma, x \in B\}$ is bounded.

Theorem 4.3. Suppose that $X$ is of second category and metrizable. If $Y$ is a locally convex space with the dual $Y^{\prime}$ and $\Gamma \subset \mathscr{W}_{\gamma, U}(X, Y)$ is pointwise bounded and $y^{\prime} \circ f: X \rightarrow \mathbb{C}$ is bounded whenever $y^{\prime} \in Y^{\prime}$ and $f \in \Gamma$, i.e., for every bounded set $B \subset X, \sup _{x \in B}\left|y^{\prime}(f(x))\right|<+\infty$ for all $y^{\prime} \in Y^{\prime}, f \in \Gamma$, then $\Gamma$ is uniformly bounded on each bounded subset of $X$.

Proof. Let $y^{\prime} \in Y^{\prime}$. Since $y^{\prime} \circ f \in \mathscr{L}_{\gamma, U}(X, \mathbb{C})$ is bounded whenever $f \in \Gamma$, each $y^{\prime} \circ f: X \rightarrow \mathbb{C}$ is continuous by Lemma 3.2. Then the desired follows from Theorem 4.2 .

Theorem 4.4. Suppose that $X$ is of second category and $Y$ is a locally convex space with the dual $Y^{\prime}$. Let $\left\{f_{n}\right\} \subset \mathscr{W}_{\gamma, U}(X, Y)$ such that each $y^{\prime} \circ f_{n}: X \rightarrow \mathbb{C}$ is continuous for all $y^{\prime} \in Y^{\prime}, n \in \mathbb{N}$. If $\lim _{n} f_{n}(x)=f(x)$ exists at each $x \in X$, then $f \in \mathscr{W}_{\gamma, U}(X, Y)$ and $f$ is continuous.

Proof. Let $y^{\prime} \in Y^{\prime}$. Since $\left\{y^{\prime} \circ f_{n}\right\} \subset \mathscr{L}_{\gamma, U}(X, \mathbb{C})$ is a sequence of continuous functions such that $\lim _{n} y^{\prime}\left(f_{n}(x)\right)=y^{\prime}(f(x))$ for all $x \in X, y^{\prime} \circ f \in \mathscr{L}_{\gamma, U}(X, \mathbb{C})$ and $y^{\prime} \circ f: X \rightarrow \mathbb{C}$ is continuous by Theorem 3.2. But $y^{\prime} \in Y^{\prime}$ is arbitrary so $f \in \mathscr{W}_{\gamma, U}(X, Y)$ and $f$ is continuous by Corollary 4.1 .

\section{References}

[1] J. L. Kelley, General Topology, D. Van Nostrand Company, Inc., Toronto-New YorkLondon, 1955.

[2] G. Köthe, Topological Vector Spaces I, Springer-Verlag New York Inc., New York, 1969.

[3] R. Li, J. Chung, and D. Kim, Demi-distributions, to appear.

[4] R. Li and C. Swartz, Spaces for which the uniform boundedness principle holds, Studia Sci. Math. Hungar. 27 (1992), no. 3-4, 379-384.

[5] R. Li, S. Wen, and L. Li, Demi-linear analysis IV, to appear.

[6] R. Li and S. Zhong, A new open mapping theorem, to appear.

[7] J. Liu and Y. Luo, A resonance theorem for a family of $\alpha$-convex functionals, J. Math. Res. Exposition 19 (1999), no. 1, 103-107.

[8] O. Naguard, A strong boundedness principle in Banach spaces, Proc. Amer. Math. Soc. 129 (2000), 861-863.

[9] W. Roth, A uniform boundedness theorem for locally convex cones, Proc. Amer. Math. Soc. 126 (1998), 1973-1982.

[10] C. Swartz, The evolution of the uniform boundedness principle, Math. Chronicle 19 (1990), 1-18.

[11] — A uniform boundedness principle of Pták, Comment. Math. Univ. Carolin. 34 (1993), no. 1, 149-151.

[12] , Infinite Matrices and the Gliding Hump, World Scientific Publishing Co., Inc., River Edge, NJ, 1996.

[13] A. Wilansky, Topology for Analysis, John Wiley, 1970. 
[14] , Modern Methods in Topological Vector Spaces, McGraw-Hill International Book Co., New York, 1978.

[15] S. Zhong and R. Li, Continuity of mappings between Fréchet spaces, J. Math. Anal. Appl. 311 (2005), no. 2, 736-743.

RONGLU Li

Department of Mathematics

Harbin Institute of Technology

HARBin, 150001, China

E-mail address: rongluli@yahoo.com.cn; rongluli@eyou.com

SHUHUI ZHONG

Department of Mathematics

Harbin Institute of Technology

Harbin, 150001, China

E-mail address: zhshuhui@yahoo.com.cn

LINSONG Li

Department of Mathematics

YANBIAN UNIVERSITY

YANJI, 133002, CHINA

E-mail address: lils@ybu.edu.cn 